Elin K. Jacob - School of Library and Information Science, Indiana University Bloomington

Guo Zhang - School of Library and Information Science, Indiana University Bloomington

\title{
The role of virtual boundaries in knowledge sharing and organization
}

\begin{abstract}
The concept of boundary is essential to investigations of knowledge organization and information sharing in the virtual world. With information and communication technologies playing an increasingly significant role in today's networked society, the idea of a boundary can no longer be tied to notions of geographical locale or intellectual domain. After reviewing traditional approaches to space and place, two concepts closely related to boundaries, we propose that it is the notion of place that both generates boundaries and provides for recognition of and distinctions between knowledge domains. In the online world, however, traditional concepts of space and place, and thus traditional understandings of boundary, are inadequate for explaining virtual boundaries because of the lack of physical embodiment in the digital environment. Rather, it is the individual's personal sense of belonging that creates an awareness of domains and facilitates the sharing of knowledge. The lack of embodiment in cyberspace shifts responsibility for the creation and recognition of boundaries to the individual, indicating that the idea of place in cyberspace -- "cyberplace" -- would best be characterized by a sense of "place-like" that is at once stable yet fluid, consistent yet dynamic.
\end{abstract}

\section{Introduction}

Zerubavel (1991) observes that the process of lumping and splitting is an inherent aspect of the human psyche: We strive to impose regularity and stability on the disorderliness of the physical world by divvying up the "things" in our environment -by constructing boundaries between groups of "things" so as to create "islands of meaning." We separate animals from plants, the lesser animals from humans, dogs from cats, and schnauzers from poodles; we impose non-natural divides between contiguous geographical spaces and designate them as countries, as states or provinces within countries, or as counties or districts within states; and we create boundaries between formal education and practical knowledge, between the sciences and the humanities, between biology and physics, and even between various specialties within the study of biology or physics. By establishing a boundary around a set of like things, we distinguish them from unlike things and create a meaningful grouping to which we can then assign a name. This name serves as a surrogate for each member of the group, for the group as a whole, and for the meaning or understanding that we ascribe to the group. By creating boundaries, we can accumulate knowledge about a named group; we can apply that knowledge to distinguish between different groups; and we can compare our knowledge of a particular group with what we know of other groups to identify differences or recognize patterns. Boundaries help us to organize our knowledge of the world around us and to develop a shared vocabulary that facilitates the transfer of knowledge.

The concept of boundary is not new to information science (IS); but its use has generally relied on the assumption that a boundary is a geographical or metaphorical line that contains a physical area by indicating its perimeters or marks the separation between two or more conceptual spheres. For example, Star and Greisemer (1989) 
propose the use of boundary objects as a means for building cooperation and sharing knowledge across heterogeneous communities involved in scientific research. However, there has been little effort to develop a comprehensive understanding of the concept of boundary or how boundaries function in IS. With the virtual world playing an increasingly significant role in today's networked society, the idea of boundary can no longer be limited to geographical locales or intellectual domains.

As the digital environment becomes ever more pervasive and more intrusive, the "traditional bounds posed by the constraints of space and time are fast being changed, in scale and scope, qualitatively as well as quantitatively" (Batty, 1997, p. 337). Accordingly, we introduce the virtual dimension of boundary as a complement to more traditional understandings of boundaries as physical or intellectual: While the virtual dimension of boundary embraces certain of the more traditional characteristics of physical and intellectual boundaries, the unique nature of cyberspace imbues virtual boundaries with a distinctive role in online practices of knowledge organization and information sharing.

\section{Boundaries in the offline world: Origins, definitions, and characteristics}

To better understand the role of boundary in knowledge sharing and organization, we propose a framework for analyzing the functionality of boundaries. This framework consists of three dimensions: the physical dimension, the epistemological dimension, and the virtual dimension, which are summarized in Table 1.

Table 1. A three-dimensional framework of boundaries

\begin{tabular}{|l|l|l|}
\hline Dimension & Origin & Operationalization \\
\hline Physical & Space and place & e.g., Communities \\
\hline Epistemological & Mental models & e.g., Categorization and classification \\
\hline Virtual & $\begin{array}{l}\text { Space and place; mental models in } \\
\text { the digital environment }\end{array}$ & $\begin{array}{l}\text { e.g., Online communities; interoperability across } \\
\text { domains and technologies }\end{array}$ \\
\hline
\end{tabular}

A boundary is commonly understood as a means for dividing geospatial configurations into spaces and places. Although philosophical disagreements continue over the distinction between spaces and places (Zhang \& Jacob, 2012a), the concept of boundary is frequently advanced as a basis for understanding differences between the complementary concepts of space and place. Since Aristotle, there has been a tendency to regard space as an unbroken and static geographic extension that is independent of human consciousness. This perspective emphasizes the dichotomy between space and place in line with other, more traditional dichotomies: objectivity and subjectivity, body and mind, materiality and spirituality.

Researchers such as Erickson (1993), Harrison and Dourish (1996), and Maglio, Barrett and Farrell (2003) contend that place is not simply a subset of space, focusing, instead, on space as a physical entity and place as a social construct. They propose that there the distinction between space and place is significant: Space is a natural fact, and place is a cultural product. Following Aristotle, these researchers regard space as the material dimension of physical things. For example, Harrison and Dourish (1996) suggest that "space is the structure of the world; it is the three-dimensional environment, 
in which objects and events occur, and in which they have relative position and direction" (p. 68); and they argue that place is "a space which [sic] is invested with understandings of behavioural appropriateness, cultural expectations, and so forth. ... Furthermore, 'places' are spaces that are valued" (p. 69; emphasis in original).For these researchers, then, space is a natural fact: a collection of properties defining the essential reality of settings of action (Dourish, 2006). As Malpas (1999) observes, the notion of space "has come to be associated with a narrow concept of physical extension" (p. 27) while place is viewed as a cultural and social phenomenon that indicates "the human response to physical surroundings or locations" (p. 30).

Humanistic geographers such as Yi-Fu Tuan emphasize the importance of the experiential dimension in shaping the perception of place. For Tuan (1977), a place is a time-based phenomenon created by human experience: It is the conjunction of the past, the present and the future. Echoing Tuan, Lipovac (1997) argues that "place is the present expression of past experiences and events and the expression of hopes for the future" (p. 6). Gieryn (2000) suggests that place is the medium through which social life happens and its most significant characteristic is that it is invested with meaning and value. He contends that place is imbued with the moral judgments, behaviors and practices that saturate social life; and he concludes that "place matters for politics and identity, history and futures, inequality and community" (p. 482): The longer people have lived in a place, the more rooted they feel and the greater their sense of belonging in (or to) that place. Within such a framework, a place is thought to emerge, to evolve and to eventually disappear over the course of history.

In the offline world, boundaries are generally perceived as limits on space (e.g., walls, state lines, country borders) that set off a place from the space(s) surrounding it and establish the range within which place-specific rules and conventions govern behavior. The recognition of boundaries and the accompanying sense of belonging to a "place" lead to the recognition of a domain and divide different domains from each other. But simply separating different domains as one would separate geographical territories does not explain the different schools of thought that often persist within a single domain. Rather, the boundaries that demarcate arenas of knowledge must be understood as shared models of the world, each of which is grounded within the awareness of a particular context or conceptual place -- an appreciation of the identity of that place, an attachment to its history, and a sense of community with the others who occupy that place.

This points to another important dimension of boundaries in the offline world: Boundaries are intrinsic components of human cognition. This aspect of boundaries is typically discussed in the psychological literature as the distinction between inside and outside or between "self" and "other". For example, Heider (1958) and Gilbert and Malone (1995) have described the tendency of individuals to resist or even to reject ideas and practices that diverge from those with which they are familiar. Gilbert and Malone (1995) point out that, "At birth, one inherits a national identity, a cultural and racial heritage, and a socioeconomic circumstance" (p. 33). These characteristics are not consciously adopted by the individual; rather, they are acquired through an association with place -- through the individual's experiences and interpersonal relationships which occur within a sociomaterial place and lead to the development of sets of expectations, or mental models, for the outcomes of certain behaviors, for the 
order in which events will occur, and for appropriate behavioral responses to those events (Mandler, 1979). The cognitive boundaries associated with a mental model function as constraints on the individual's understanding of events and minimize cognitive processing by limiting the range of appropriate behavioral responses from which the individual may choose. Because these cognitive models are able to reflect and to respond to an individual's ongoing interactions within the environment, they are inherently flexible, capable of restructuring existing boundaries through the integration of new experiences.

While personal, experientially-based mental models are flexible and potentially dynamic, the need to share specialized knowledge requires the imposition of cognitive constraints that can be shared across members of a community or intellectual domain. To ensure that disciplinary knowledge is consistent across individuals, the acquisition, representation and transmission of this knowledge demand a shared vocabulary that both constrains the meaning of individual concepts and establishes relationships between or among concepts. This stability of reference is achieved through the aegis of a shared language that imposes well-defined boundaries on its representations. As Foucault observes, the very possibility of introducing stability within a domain assumes that knowledge can be defined and arranged -- that knowledge is "at the same time describable and orderable" (p. 158). Because a domain vocabulary is necessarily embedded within the context of the domain, it not only establishes the intellectual boundaries of the domain but also prescribes and perpetuates a particular world view -a shared mental model -- that makes every proposition "an invariable pattern of reality" (p. 136).

\section{Virtual boundaries: From the offline world to cyberspace}

As computers and digital computation become more pervasive, "traditional bounds posed by the constraints of space and time are fast being changed, in scale and scope, qualitatively as well as quantitatively" (Batty, 1997, p. 337). This process of change has generated the dimension of space that we now call "cyberspace." Cyberspace has been characterized as a form of "virtual reality" that both "afford[s] social interaction and embod[ies] cultural values" (Kalay \& Marx, 2001, p. 770). But cyberspace has also been conceptualized as an unspecified, unruly and boundless space, a huge black void, or a simple "container" for holding "data, services, information of many kinds, as well as for talking, browsing, and for all types of communication that traditionally have taken place face-to-face" (Batty, 1997, p. 339).

One approach to an understanding of cyberspace is to view it as a spatial metaphor. Linguistically, the term "cyberspace" is the compound of "cybernetics" and "space", indicating an interdisciplinary, multi-dimensional and multi-leveled "space". In 1984, William Gibson, the author of Neuromancer, described cyberspace as "A consensual hallucination experienced daily by billions of legitimate operators, in every nation, by children being taught mathematical concepts. A graphic representation of data abstracted from banks of every computer in the human system. Unthinkable complexity" (p. 67). In 1991, Benedikt characterized cyberspace as a "parallel universe" (p. 15); in 1993, Batty described it as a "new kind of space, invisible to our direct senses, a space which might become more important than physical space itself [and which is] layered on top of, within and between the fabric of traditional 
geographical space" (pp. 615-616); and, in 1998, Graham suggested that cyberspace should be considered "a fragmented, divided and contested multiplicity of heterogeneous infrastructures and actor-networks" (p. 178).

Although such descriptions focus on two primary dimensions of cyberspace-the physical and the technological infrastructures represented by "spatial and technological metaphors" (Graham, 1998, p. 167) -- they also implicate a third, more abstract dimension: that of spiritual, social and cultural awareness -- of social practice and the human experience -- which cannot be explained through the use of spatial metaphors. Aarseth (2007) discusses the uniqueness of "spatial representation" in computer games -- a specific instantiation of cyberspace -- and its relation to "real space":

By being generated, cyber places are "regions in space" and cannot exist as parallels of real, three-dimensional space. .... "Cyberspace" and other such phenomena (e.g. computer games) are constituted of signs and are therefore already too dependent on our bodily experience in and of real space to be "hallucinated" as space. Moreover, the fact that they are not real space but rather objects and places is the only reason we can perceive them at all (pp. 44-45).

He posits that "spatial representation in computer games [is] a reductive operation leading to a representation of space that is not in itself spatial, but symbolic and rulebased" (p. 45), concluding that "computer games are allegories of space [that] pretend to portray space in ever more realistic ways but rely on their deviation from reality in order to make the illusion playable" (p. 47). His arguments are in some ways contradictory: On the one hand, he considers cyberspace a symbolic allegory of space and thus not "real space"; on the other hand, he claims that cyberspace has rules that depend on human experience, as places do, yet he argues that, although cyberspace is not a "place", it is constituted by places.

An examination of the literature indicates that traditional concepts of space and place as well as traditional understandings of boundaries are inadequate for explaining either cyberspace or virtual boundaries given the absence of physical embodiment in the digital environment. According to Waterworth, Lund and Modjeska (2003), "we are embodied beings, [and] meaning ultimately resides in bodily experiences" (p. 125). They argue that humans "have evolved to act in the physical world, and how we are able to understand abstract information is derived from that capacity. ... We experience the physical world as a three-dimensional space, with gravity holding our bodies, other people and [other] things onto horizontal surfaces" (p. 125).

Because the absence of embodiment precludes the possibility of a sensory intermediary as well as the capacity to abstract places from cyberspace, traditional models of space can be obfuscating, causing Web users to feel "lost" when surfing online. Waterworth et al. (2003) contend that, "since we all share the same evolutionary history and hence, bodily structures and potential for experiences, we share the same primitives for understanding information. This is what makes social interaction-and social navigation of information spaces-possible" (p. 125). The absence of embodiment in cyberspace precludes a shared carrier for appropriate social action and behavioral framing, both of which are essential for effective information sharing and knowledge organization in the offline world.

Physical embodiment in the sociomaterial world indicates that domain boundaries are mediated by geographical or conceptual proximity. In contrast, groups on the web are bounded by other forms of proximity: by the proximity of emotions, by the 
proximity of shared concerns, by the proximity of common goals, etc. Because web groups are initiated and maintained in a digital environment, physical proximity -physical "place" -- is neither a necessary nor a sufficient criterion for sharing knowledge. Shared commonplaces rather than physical or geographical territories are prioritized. These commonplaces -- the commonalities and conventions united by a shared boundary -- will determine whether participants constitute a domain of viirtual experience or knowledge community. Building on the forms of communication and interaction supported by the platform of the web, the emergence and evolution of an online domain or community will depend on the number of users involved and their passion rather than their physical proximity. Crossing both geographical and national boundaries, web users who share common interests, habits, values, goals or behaviors can come together to exchange information and knowledge (Zhang \& Jacob, 2012b).

Without embodied experiences in cyberspace, immersion in the digital environment may be accompanied not by a "sense of place" but by a "sense of place-like", confusing efforts to interpret cyberspace and to understand the nature of virtual boundaries. The physical world is spatial, but the world of the digital environment is a "spatial cueing world" (Waterworth et al., 2003, p. 130). In principle, then, the "space" in "cyberspace" is a metaphor that reflects an individual's three-dimensional model of the physical world: Cyberspace is viewed as a "space" because the spatial metaphor allows us to "project our spatial experiences ... to abstract, non-spatial domains of experience" (Waterworth et al., 2003, p. 139). Because cyberspace supports awareness of and interaction with others, it affords individuals the ability to gather in virtual places: "[A] place does not necessarily map to a location in web space, but might be automatically constructed based on the interests and activities of web users" (Maglio \& Matlock, 2003, p. 402). Individuals are able to "explore virtual worlds of information using cognitive processes similar to those with which they explore the real world" (Waterworth et al., 2003, p. 148). Thus cyberspace provides for the exchange of interpersonal experiences, mental models and knowledge and supports the emergence of "cyberplaces", where each such "place" is a sociocultural product "invested with understandings" (Harrison \& Dourish, 1996, p. 69).

However, the absence of embodiment in cyberspace precludes appropriate boundaries for behavioral framing of social actions: Without the possibility of threedimensional physical experiences, cyberspace cannot support the perception of direction and or the recognition of relationships: Immersed in cyberspace, we lose any sense of what is up or down -- of what is before or after, in front of or behind. More importantly, we lose the boundaries that provide behavioral framing for social interactions: The sense of boundaries established by mental models in the offline world becomes a computer- or avatar-mediated process in the virtual world, which can confound the individual's efforts to interpret meaning, to share his own mental models, or to trust the mental models of others in the virtual environment.

The virtual boundaries of cyberspace evince features that are different from those of the offline world. Cyberspace is simultaneously bounded both by the web's structure of connections and by the way the individual accesses a particular web site or web page. For the initiated, cyberspace is not an uninhabited or undifferentiated space without boundaries but a collection of possibilities and opportunities (Massey, 2005): It is a familiar and recognizable "neighborhood" imbued with personal identities, social 
interactions and a sense of belonging in a "place". The boundaries imposed by the structure of the web or by physical access to the web - the technical infrastructure of computers, wires, fibers, Wi-Fi and protocols - distinguish different place-like units in the digital environment. As Maglio et al. (2003) observe, "[u]sers are considered to be in the same place when they are currently viewing the same web page, or pages on the same web site, or pages hosted in the same domain" (p. 252).

It is just this sense of belonging to a "place" that enables the fashioning of virtual domains, and the emergence of a shared vocabulary supports the exchange of information -- of knowledge -- among domain participants. But, unlike the wellconstructed language that facilitates knowledge sharing among domain participants in the physical world, the virtual boundaries that demarcate a cyberplace and the vocabulary that expedites knowledge sharing are not static but dynamic: Participants in a cyberplace will vary across time, relationships and interactions between participants will ebb and flow, and the language of the cyberplace -- the domain -- will evolve in response to the needs of participants. Because cyberspace is fluid and dynamic, boundaries can be constructed, broken, and re-constructed; knowledge can be organized and re-organized; and inforation can be exchanged and changed and exchanged again.

\section{Conclusion}

Boundary is a central concept in the investigation of theoretical and practical issues related to knowledge sharing and organization in the virtual world. With information and communication technologies playing an increasingly significant role in today's networked society, the idea of boundaries can no longer be tied to geographical locales or intellectual domains.

In the offline world, the concept of a boundary is commonly understood as a means for dividing geospatial configurations into spaces and places. However, it is not space but the search for place -- the search for a sense of belonging -- that drives the creation of physical boundaries and underscores the social understandings associated with the boundaries of human experience. Boundaries are also intrinsic components of human cognition, dividing knowledge among various conceptual domains and promoting stability of meaning through the development of a shared vocabulary of bounded concepts.

In the online world, however, traditional concepts of space and place as well as traditional understandings of boundaries are inadequate for explaining cyberspace, cyberplaces, and the role of virtual boundaries. The "space" in "cyberspace" is a metaphor that depends on the individual's three-dimensional model of the physical world and supports exchange in the virtual world of interpersonal experiences, mental models and knowledge. However, the absence of embodiment in the digital environment imbues virtual boundaries with features that differ dramatically from boundaries in the material world: A cyberplace is simultaneously bounded not by physical demarcations but by the web's structure of connections and the way the individual accesses a particular web site or web page. More importantly, the lack of embodiment an individual experiences in cyberspace shifts responsibility for both the creation and the recognition of boundaries onto the individual himself: the sense of belonging to (or in) a place that characterizes the individual's recognition of place in 
the material world is replaced by a sense of place-like belonging that is at once stable yet fluid, consistent yet dynamic. Thus a clearer understanding of the various dimensions of boundaries and their diverse functions -- an understanding that is not tied to the physicality of the material world -- will contribute a significant dimension to studies of knowledge organization and knowledge sharing in the digital environment.

\section{References}

Aarseth, E. (2007). Allegories of space: The question of spatiality in computer games. In F. V. Borries, S. P. Walz \& M. Böttger (Eds.), Space time play computer games, architecture and urbanism: The next level (pp. 44-55). Basel: Birkhäuser Verlag

Batty, M. (1997). Virtual geography. Futures, 29(4/5), 337-352.

Benedikt, M. (1991). Introduction. In M. Benedikt (Ed.), Cyberspace: Firststeps (pp.1-18). Cambridge, MA: MIT Press

Dourish, P. (2003). Where the footprints lead: Tracking down other roles for social navigation. In K. Höök, D. Benyon \& A.J. Munro (Eds.), Designing information spaces: The social navigation approach (pp. 273-291). London: Springer-Verlag.

Gibson, W. (1984). Neuromancer. New York: Basic Books.

Graham, S. (1998).The end of geography orthe explosion of place?Conceptualizing space, place and information technology. Progress in Human Geography, 22(2), 165-185.

Erickson, T. (1993). From interfact to interplace: The spatial environment as medium for interaction. In Proceedings of the Conference on Spatial Information Theory (pp. 391-405). Heidelberg: Springer-Verlag.

Gieryn, T. F. (2000). A space for place in sociology. Annual Review of Sociology, 26, 463 496.

Gilbert, D. T., \& Malone, P. S. (1995). The correspondence bias. Psychological Bulletin, 117(1), 21-38.

Harrison, S., \& Dourish, P. (1996). Re-place-ing space: The role of place and space in collaborative systems. Proceedings of the 1996 ACM conference on Computer supported cooperative work (pp. 67-76). New York: ACM Press.

Heider, F. (1958). The psychology of interpersonal relations. New York: Wiley.

Kalay, Y. E., \& Marx, J. (2001). The role of place in cyberspace. In Proceedings of the Seventh International Conference on Virtual Systems and Multimedia. October 25-27, Berkeley, CA. Washington: IEEE Computer Society.

Karakas, F. (2009). Welcome to World 2.0: The new digital ecosystem. Journal of Business Strategy, 30(4), 23-30.

Lipovac, N. (1997). Space and place. Prostor, 1(13), 1-34

Maglio, P. P., Barrett, R., \& Farrell, S. (2003). WebPlaces: Using intermediaries to add people to the web. In K. Höök, D. Benyon, \& A.J. Munro (Eds.), Designing information spaces: The social navigation approach (pp. 249-272). London: Springer-Verlag.

Maglio, P. P., \& Matlock, T. (2003). The conceptual structure of information space. In K. Höök, D. Benyon, \& A. J. Munro (Eds.), Designing information spaces: The social navigation approach (pp. 385-403). London: Springer-Verlag.

Mandler, G. (1979). Organization and repetition: Organizational principles with special reference to rote learning. In L. G. Nilsson (Ed.), Perspectives on memory research (pp. 293 327). Hillsdale, N. J.: Erlbaum. 
Malpas, J. E. (1999). Place and experience: A philosophical topography. Cambridge: Cambridge University Press.

Star, S. L., \& Greisemer, J. R. (1989). Institutional ecology, 'translations' and boundary objects: Amateurs and professionals in Berkeley's Museum of Vertebrate Zoology, 1907-39. Social Studies of Science, 19(3), 387-420.

Tuan, Y. F.(1977). Space and place: The perspective of experience. Minneapolis: University of Minnesota Press.

Waterworth, J. A., Lund, A., \& Modjeska, D. (2003). Experiential design ofshared information spaces. In K. Höök, D. Benyon, \& A. J. Munro (Eds.), Designing information spaces: The social navigation approach (pp. 125-149). London: Springer Verlag.

Zerubavel, E. (1991). The fine line: making distinctions in everyday life. Chicago: University of Chicago Press.

Zhang, G., \& Jacob, E. K. (2012a). Reconceptualizing cyberspace: "Real" places in digital space. The International Journal of Science in Society, 3(2), 91-102.

Zhang, G., \& Jacob, E. K. (2012b). Community: Issues, definitions, and operationalization on the Web. In Proceedings of the World Wide Web Conference Companion, 2012 (WWW 2012) (pp.1121-1130). April 16-20, Lyon, France. New York: ACM. 